\title{
Early stimulation of the amputed member patient lower \\ Introduction
}

The general incidence of amputations steadily rises due to the higher accident rates and the average prolongation of life that allows the survival of elderly people with general disorders that predispose to amputation (such as peripheral ischemia). Thus, KESSLER indicates that, during World War II, 18,000 American soldiers lost members as a result of war injuries, but in the same four-year period, 120,000 civilian Americans were amputated as a result of accidental injuries. ${ }^{1}$ In the United States, 1 in 200 Americans has experienced a major amputation; every year there are 35,000 amputations due to congenital defects and general causes, being the most frequent location in the lower extremities (10.3 in relation to the upper extremity). In England and Wales there are more than 80,000 people who have lost one or more members: 27,000 due to wars and 60,000 due to injuries or illnesses. Approximately 3,500 new cases are sent to the 21 national prosthetic centers each year.

The proportion of amputations in men is 2.5 times higher than in women. Of the new cases produced, $27 \%$ are due to accidents, $67 \%$ due to illness and the remaining $6 \%$ due to congenital deformities. Half of the amputees that are currently produced are older than 60 years old (RITCHIE). ${ }^{1}$ In Japan, the frequency of amputations due to work-related accidents is 3.4 times higher than in the United States, and the proportion of amputees of the upper extremity is 14 to 10 in relation to those of the lower extremity (IrnA). The statistical studies carried out by GLATTLY have allowed (in the United States) to obtain the following conclusions:

1. Amputations due to accidents in men are nine times more numerous than in women, due to the higher risk activities that man develops.

2. Amputations in men due to disease are 2.6 times more frequent than in women.

3. The frequency of amputations due to tumors is very similar in both sexes.

4. Congenital deformities of extremities are of similar frequency in both sexes.

5. There is no difference in incidence in right or left members.

6. The greatest frequency of amputations due to traumatism is found in the decade from 41 to 50 years; due to illness, between 61 and 70 and, due to tumors, between 11 to 20 years. In Spain, amputees are estimated at 50,000.1

\section{Causes of amputation}

RUSK establishes the following classification of amputations from the etiological point of view

1. Accidental injuries (traffic accidents $-63 \%$, with greater incidence in lower extremities-, industrial accidents -

2. 73 to $81 \%$, with higher incidence in upper extremities-)

3. Peripheral vascular diseases (tissue death due to arteriosclerotic or diabetic peripheral vascular insufficiency).
Volume II Issue 6 - 2018

Anicia Hernández Soublett

National Institute of Angiology and Vascular surgery, Cuba

Correspondence: Anicia Hernández Soublett, National Institute of Angiology and Vascular surgery, Cuba, Email aniciandez@infomed.sld.cu

Received: October 26, 2017 | Published: December 05, 2018

4. Diseases of peripheral vessels of small caliber, Buerger and Rainaud.)_?

5. Malignant neoplasms. Long-lasting infections of bones and other tissues that do not allow the restoration of

6. function (TB, gangrene, osteomyelitis).

7. Thermal injuries.

8. Useless deformed limb that the patient considers unsightly.

9. Uncited states that may endanger the life of the patient, such as a vascular accident or snake bite.

10. Congenital lack of member.

\section{Rehabilitation}

\section{Consensus for amputation level}

Taking into account the optimal level of amputation is essential when we visualize the expectations of rehabilitation trying to minimize the invalidating signs as much as possible. In the specific case of the amputation of a limb, the need for surgical-rehabilitative coordination becomes more evident. A level of collegial amputation between these two disciplines can be the cornerstone to achieve a successful prosthetic treatment. Disability disorders added to the amputation, complicate the rehabilitation possibilities of the amputee MURPHY has studied very aggressively the aggravating factors that can transform the simple amputation into a much deeper disability. Among them, due to their greater importance, the following can be highlighted. ${ }^{2}$

\section{Neurological disorders}

\section{Paralysis is factors that hinder rehabilitation}

The paralysis or resections of the stump muscles affect the control over the prosthesis of other parts of the body, influence the type of walking, action of the straps, manageability to place or remove the prosthesis and alteration in the use of canes. Paraplegia and hemiplegia present special problems not only for the type of existing paralysis, but also for the alterations in sensitivity. Certain pyretic or paralytic conditions suppose the ease for the bone fracture of the stump, which will complicate the adaptation of the prosthesis or decrease the effective bone level. ${ }^{2}$ 


\section{Sensory disorders}

Vestibular dysfunctions due to disease or drug toxemia can cause alterations in balance. Bodily imbalance becomes dangerous in amputees of lower extremities, especially when walking in the dark in vehicles (boats, trains, etc.). ${ }^{2}$ Deafness, especially of high frequency sounds, can incapacitate the amputee to perceive the sounds of his prosthesis (such as the knocking of the knee) and constitute a social difficulty. In these cases, it is advisable to recommend greater attention and vigilance in the care and lubrication of prostheses. They should also be warned of the dangers of a sudden collision or stoppage due to lack of knowledge of a danger reported by hearing, which can make you lose your balance (especially on the street, when passing in front of a vehicle exit). ${ }^{2}$ Sometimes, vision disorders can be a manifest difficulty for this class of patients. Thus, a small refractive error can cause the edges of a step to become out of focus. Also the alteration of the capacity of perception of the depth usually is cause of stumbles. The blind amputee of the lower extremity walks more safely if the short cane is used. ${ }^{2}$ The anatomical clamp, according to the KRUKENBERG technique, has a specific and exclusive application in the blind. The loss of sensations of proprioception and sensitivity constitutes a serious complication in the amputee, who not only must depend very closely on the sight to use his prosthesis, but also can easily present macerations or ulcerations in the stumps. This type of disorder is found in paraplegics, syringomyelics, multiple sclerosis, leprosy, or individuals with peripheral circulation deficits in whom a stump has been left too long. ${ }^{2}$

\section{Generalized physical disorders}

Sometimes, various processes, generalized diseases can hinder the use of prostheses. Some, such as Buerger's disease, arteriosclerosis and diabetes, may not only require amputation, but also seriously affect the function or decrease the physical capacity of the amputee. Both the patient and the therapist should monitor in these cases the presentation of irritations of the stump, since their healing, once produced, is very slow. Also, the existence of edema in some of these processes complicates or perhaps prevents the use of suction lace. ${ }^{2}$ Occasionally, heart disease limits physical performance, so the exercise plan must be of a very conservative design for the patient's therapeutics. The fear of the claudication of the knee acts as an intense stress on the heart; therefore, more stable joints or the use of two rods are recommended. ${ }^{2}$ Skin conditions in the areas of lace or straps may pose temporary difficulties for adaptation. Sometimes, these alterations of the skin are secondary to misfit adaptation. Likewise, osteomyelitis of the stump is a contraindication for prosthetic adaptation. ${ }^{3}$

\section{Psychological factor}

1. Low intelligence or poor neuromuscular coordination can hinder teaching or complicate adaptations.

2. Unrealistic attitudes, lack of motivation and a depressive morality are factors that manifestly damage recovery. ${ }^{3}$

3. Anatomofunctional alterations.

4. Jointly or independently with the complications inherent to the surgical practices are others, the stump can

5. Present physical or functional alterations that suppose a complication.

The degeneration of the stump occurs with very different degree in all cases. Degenerative alterations can be observed in muscle or residual muscle fragments, especially atrophy of muscle fibers accompanied by proliferation of myolysis, disintegration and fatty infiltration. These disorders are caused by anatomical damage during surgery, scarring and atrophy due to denervation. The vascular contraction reflects, also influences the atrophy. The appearance of ring myofibrils around muscle fibers is also characteristic (APATENKO) Progressive atrophy can make the fittings of the prosthesis useless, which need to be modified or filled in constantly. Myoplastic techniques for amputation considerably reduce this danger.

Muscle contractures as a complication, are characteristics of poor care of the stump. Many times its presentation

will suppose the absolute failure of all the prosthetic possibilities. They are usually avoided if the patient has been subjected to specific postural and kinesitherapeutic care very early. ${ }^{1}$ Circulatory disorders of the stump occur as a consequence of peripheral vascular disease, poor adaptation of the prosthesis, sustained pressures, ulcerations and congestive dermatitis or poor hygiene. The treatment will be causal, not forgetting the practice of active exercises by the patient.

Dermal disorders can constitute a dangerous "handicap" for the use of prostheses, because the diagnosis and treatment of these conditions must be very early. Edema, contact dermatitis (due to gum-like substances, plastics, lacquers, resins, etc.), epidermoid cysts, pyoderma (folliculitis and furunculosis), fungal infection, intertriginous dermatitis, chronic ulcers, warty hyperplasia, tumors and cases are frequent. Special acne, seborrheic dermatitis, eczema and psoriasis. Acute hygiene will be practiced using soaps with hexachlorophene. It is convenient to carry out this washing at night without forgetting the daily cleaning of the prosthetic socket. Neither will the monitoring of the pressures on the stump be forgotten. ${ }^{1}$

Painful syndromes can be one of the worst complications of the amputee, from his first appearances. Where the patient under the influence of discomfort neglects postural care and reduces his disposition towards aspects related to rehabilitation. ${ }^{1}$ The low back pain that occurs in some unilateral lower extremity amputees is not a serious complication since it usually does not appear during walking, but rather in the somewhat prolonged static standing. In general, it is motivated by vertebral alterations due to the use of a short prosthesis. Occasionally, phantom pain or painful phantom limb is an almost completely disabling complication, and may occur in any amputated limb.

In the study by Christopher and Koepke it was shown that in $10 \%$ of the cases of painful phantom limbs in amputees of the lower limb, the cause was the entrapment of the nerves sectioned by other scar structures. It is assumed that this syndrome can be initiated by peripheral irritation of these sectioned nerves, which stimulate the superior structures, but, in the absence of anatomical coexistence, it becomes pain. The description of phantom sensation and stump pain serve to identify the nerve, as well as skin reaction, Tinel's sign and hyperesthesia. The electromyographic and chronaximometric exploration of 20 amputees showed that the affectation of the coordination and proprioception for the abolition of isometric contractions can be a cause of the phantom limb. But these phenomena do not occur if the amputation took place before 4 years of age. The incidence begins to appear and rise above 8 years.

The stump pains, according to LERICHE can be spinal, vasomotor, causal and mixed, but whatever their origin, the conservative therapeutic procedures are of very problematic efficacy, 
use of vitamins, anesthetizing infiltrations of the sympathetic nerve, intra-arterial or intravenous injections of novocaine, infiltrations of nerve trunks with novacaine, radiotherapy with X-rays, subarachnoid injection with absolute alcohol, etc. The practice of psychotherapy and progressive relaxation exercises, together with the correction of prosthetic defects originates, sometimes, an improvement of the painful syndromes. ${ }^{4}$

Dederich proposes several surgical approaches for the intense pain of the stump; periarterial sympathectomy, resection of the obliterated artery and sympathetic ganglion radicotomy, interventions aimed at the sensory tracts, terminal neuroma resection, neurotomy, posterior radicotomy, White tractostomy or mesocephalic level of Dogliotti, mid-commissure myelotomy, infiltration with novocaine centers Clovis cortices and prefrontal leucotomy. In general, the result of these interventions is reserved criteria. Frequently obliteration of the nerve is easy and relatively effective, as effective can be sympathectomy and thalamotomy (Spiegel, Wyzies, Riechert). Occasionally, the pain persists even after a hemisphere is removed and despite the body hemianesthesia produced. Sometimes, the pain is due to a circulatory failure because the stump is not functional and the anchoring of the muscle groups has not been practiced, as is very often seen with the adductors in thigh amputations. In short, it would be an anoxemic stress. The myeloplastic techniques between antagonist muscle groups of the stump constitute an excellent prophylactic means. The construction muscle of the stump is a physiological method to maintain in the best conditions all the anatomical elements, muscles, bone, vessels and nerves, considerably facilitating the circulation as can be verified by arteriography. Although pain is usually rare in amputees, specific problems such as ossification, periosteal growth and loss of muscle growth can arise. The stump shows a tendency to remain small as the child grows, especially in amputations of the femur. $^{5}$

\section{The ghost member}

Karaguiosov, Krusen, Jensen and Wall establish that an exact distinction must be made between three separate entities according to the type of sensation that make up the phantom limb phenomenon in stump pain, phantom sensation and phantom limb pain ${ }^{5}$ The pain of the stump is a specific pain and located in a visible place the ghost sensation is, everything that the patient refers making mention of a segment of his limb that no longer exists and therefore is invisible, We can then easily conclude that the Phantom pain is the pain that refers in that non-existent segment. Phantom limb pain is a painful manifestation that is apparently localized in the part of the body that is missing. ${ }^{5}$ There are two main groups of theories about their cause; the psychological and the organic. The latter, in turn, is subdivided into peripheral, central and mixed.

Psychological mechanisms alone are not the answer, but they play a contributing role. The phenomenon is often triggered by emotional disturbances and is sometimes abolished by distraction, conditioning, hypnosis and psychotherapy, or it can serve as a defense against a threatening new reality and the sensations help to deny the loss and preserve a sense of physical integrity and psychological Peripheral organic theory holds that the nerve endings in the stump generate impulses that the central nervous system receives as painful. ${ }^{5}$

It has been shown that neurons produce an advancing concentration of afferent impulses in both myelinated and unmyelinated fibers. ${ }^{5}$ The primary central organic mechanism constitutes the gate control theory.
According to this theory, a portion of reticular formation of brain stem acts as a central regulatory mechanism, exerting an inhibitory or buffering effect in the reception of messages from sensitive nerves of a distant part. When many sensory fibers are destroyed by amputation of a limb, the magnitude of the entry into the reticular formation decreases. The result is that the inhibitory influence also decreases. The net effect is a kinetic activity, self-sustains at all neuronal levels and can be triggered repeatedly by the remaining fibers. Pain occurs when the output of self-sustaining sets of neurons reaches certain level or exceeds it. ${ }^{5}$ The mixed organic theory invokes the participation in the generation of the image of the missing limb as well as peripheral and spinal mechanisms. ${ }^{5}$

\section{Characteristics}

The pain in the stump is localized, as of friction, differentiating itself from that produced by local injury, sepsis, claudication or diffuse from shock and stimulation. It is more common in traumatic amputees than vascular ones in those who do not use prostheses frequently and is thought to possibly cause phantom limb pain. The phantom sensation (the perception of the missing member in variable attitudes of length and position) has on the perceptions that are expected; tingling, numbness, pruritus, heat, cold, heaviness or lightness. They are more notable in the distal parts due to their greater mobility and the high degree of sensitivity. It is greater in the dominant member; it can appear from the first 24 hours and gradually diminish although there is no clear evidence that it ever disappear completely. ${ }^{6}$

Phantom limb pain occurs after an amputation or extensive avulsion has healed. It is more common because of traumatic cause. The distal portion of the limb is the one that mainly participates. The types of pain are described as stitches, intense electric current, pins, burns, pressure, cramps, crushing, pulsation, pruritus, cutting, tearing and pricking. The higher the amputation level, the more likely it is that it will occur. The onset is variable, can last a short time or at intervals for years. Many factors can cause or aggravate pain: manipulation of the stump in the immediate postoperative period, urination, defecation, ejaculation, exposure to cold, angina pectoris, herpes zoster, bursitis, herniated disc, multiple sclerosis, prostatitis, seizures, emotional stress, changes in weather, night hours, ischemia by gauge cuff, spinal anesthesia, defective prosthesis adjustment and lack of use of it.

\section{Treatment}

There are multiple treatment alternatives and only $30 \%$ are currently used as a conservative treatment, which has provided the greatest relief. Separated into subgroups; medicated, anti-inflammatory, analgesic, vitamin, infliltrative; with anesthetics, steroids in the stump, nerve trunks and spinal canal, postural physiotherapy, compression bandage, massage therapy, kinesiology, thermotherapy, hydrotherapy, ultrasonotherapy, electrotherapy, magnetic therapy, prosthetic treatment, traditional: acupuncture and peloids, psychotherapy, occupational therapy, surgical, exeresis of hypersensitive scar and neuromas, stump regularization or reamputation, transections at different levels of the Central Nervous System. ${ }^{6}$

\section{A correct stump}

In order to achieve effectiveness and obtain the correct stump after surgery, it is necessary that in advance of the same there is identification between the surgical team and the physiotherapist that 
achieves a consensus with the patient about the level of amputation consistent with the aspirations of a patient.

\section{Prosthetic treatment}

Anatomodynamic basis will determine the effectiveness of future loss function. It is the physical structure for the placement of the prosthesis and the essential active element that will mobilize it in a conscious, oriented and coordinated manner. Therefore, any difficulty in the execution of a movement, either for reasons of blockage to the movement or lack of power for its optimal development, will constitute a serious complication that can nullify.

1. The objectives of the preparation of the stump by Gullicks0n

2. Keep the angle of movement in normal limits.

3. Prevent, or correct, if presented, muscle retractions. . Avoid or correct alignment defects.

4. Improve the circulation and nutrition of the stump. Establish muscle balance

5. Restore or increase muscle strength, endurance and coordination.

6. Prevent excessive tissue atrophy. Maintain and improve neuromuscular reactions. Maintain and improve neuromuscular reactions.

7. Many of the purposes that we have just indicated can be covered by an adequate and directed postural treatment of the patient from the moment the surgical and constructive intervention of the stump ends.

Amputees of the lower extremities, which are the most severely affected by poorly directed postural positions, can be fitted with corrective traction while remaining in bed in the healing phase. This traction of superficial tissue guides the stump toward the center of the bed (in the supine and prone positions) while displacing the soft tissues of the lining toward the apex of the stump. Likewise, from the first moment, the patient will be stimulated to change his decubitus from supine to prone by prohibiting the amputees of the lower limbs to stay in lateral decubitus, since this is a favorable position for hip flexions. The pads will also be prohibited to keep the stump flexed and the stays very prolonged in chair, especially in the double amputees. The patient will be taught, during his stay in bed, to keep the pelvis well balanced and not lateralized, for which the amputee himself will control with his hands the position of his iliac crests and relax the muscle groups on the elevated side. From the first moment (48 hours after the intervention), passive mobilizations of the stump and isometric contractions of the same will be repeated several times a day. It should not be forgotten that muscular contractures arise, generally, in a very immediate stage after surgery.

\section{Justification}

Faced with a health problem as sensitive as it turns out to be for an individual the loss of a limb. We must look for each person in his discipline some blind spot between what is still controversial today. According to the existing literature, the mechanisms that trigger phantom stump pain are not defined; some authors are based on psychological factors where emotions play their role and / or in organic reasons. Let's reflect on an individual who never considered how to move, nor to learn to walk again, an individual with plans, dreams, goals and suddenly has to see the world from another perspective. You need to internalize your loss and accept your new health condition.

\section{The physiotherapist}

Depending on the protocols of each institution, there will be an opportunity for the intervention of the physiotherapist. In our particular case we reach the patient before, the amputation, where in advance there is a relationship with the surgical team. Our work is to psychologically support the patient and incorporate it in the first 48 hours after the surgical act (sitting in bed) we do it with the use of some canvas bands that, we link to the foot of the bed, we only hold the patient by the nape of the neck and they as if using a harness are sitting down to see for the first time the result of the surgical act. We have verified that it is the first step towards acceptance.

\section{The theoretical basis}

1. The therapy of acceptance and commitment is part of the discovery of new forms of learning in human beings.

2. Psychopathology in acceptance and commitment therapy and its treatment.

3. For the therapy of acceptance and commitment, psychopathology, that is, the additional suffering inherent in life, appears when there is a lack of psychological flexibility, that is, when we do not have the capacity or do not know how to freely choose the behavior we want to perform in a given context.

The psychological processes that produce psychological rigidity are:

1. Experiential avoidance. That is, not wanting to experience the thoughts, memories, feelings, sensations and emotions that appear in a certain context.

2. Do not live in the present. The thought takes us to the future or to the past and we can be living in what our thoughts reproduce us, instead of living in the present that is where our life really takes place.

3. Lack of clarity with one's values. Our thoughts tell us what direction we want to take in life, that is, where we want to direct our behavior. The desired long-term consequences are the values that set the direction we want to follow. When we have not defined those directions or do not follow them for whatever reason, psychological rigidity occurs.

4. The lack of commitment and determination to follow one's own values and interests.

5. Merging with the content of our "I" instead of living our "I" as a context. The defense over our interests of a concept of ourselves, that is, of our SELF, instead of being aware that we are the context in which our experiences are given and that, from this point of view, we transcend our thoughts, feelings, sensations and emotions.

6. The fusion with our thinking. When what marks our behavior above our interests and what the context tells us is what conditions our behavior, it is when

It produces a fusion with our thinking that produces psychological rigidity. These processes are all interconnected so that, for example, the fusion with thought is present in the merger with the I as content, prevents living the present, away from our values, and is a component of the avoidance of experience. The goal of acceptance and commitment therapy is to eliminate psychological rigidity for what 
proposes the practice of basic processes that enhance psychological flexibility; acceptance, living the present, definition and commitment to freely chosen values, living the experience of the self as a context and the deactivation of thought (cognitive defusion). These processes, like those that produce rigidity, are totally related (Hayes et al, 2004).

The basic processes that lead to psychological flexibility are:

1. Acceptance: open to feel unpleasant emotions to focus on achieving the objectives and values present in the situation. It is not resigning or enduring; but focuses on what you can control and stop trying to control what you cannot.

2. Dismantling of thought (defusion) (Luoma and Hayes, 2003). It is about taking distance and not following them without taking the necessary time to reflect and act in accordance with the objectives that are being pursued at that time.

3. Live the present. Bringing attention to what is happening and feeling at that moment, which allows us to act more efficiently.

4. Choose our values. Choosing the desired long-term consequences in which we will invest most of our efforts Commitment to our values.

5. Live the ME as context. We are the context in which our private processes occur. $^{8}$

\section{Development}

We designed a canvas device to assist the incorporation of the amputee patient in the first 48 hours of the surgical act with the general objective of contributing with the early elements of rehabilitation the acceptance of their new physical state and stimulating postural care, alignment, balance and also avoid cardiovascular disorders due to prolonged bed rest. Our point of reference was the steel trapeze, of German manufacture whose approximate market value is 90.95 per unit; our device has a manufacturing value of 47 pesos.

\section{Universe}

We apply this procedure to all patients who undergo major amputation of the lower limb who are hospitalized in our center and who, upon prior information, are in mutual agreement with the practice. In addition, the relative is instructed on the benefits and the help that can be provided in their rehabilitation.

\section{Results}

The acceptance of the use of the device is unanimous and more than $60 \%$ of our graduated patients achieve independence for transfers from the bed to the chair and vice versa, perform balance exercises in sitting position and early activities of daily life.

\section{Conclusion}

The physiotherapist's work with the amputated patient must work from the perspective of an early stimulation, influencing from the psychological to the physiological sphere. Man can sometimes move his emotions according to the plane from which he is looking at life. Lying on a bed can make a difference.

\section{Acknowledgments}

None.

\section{Conflicts of interest}

Author declares that there is no conflicts of interest.

\section{References}

1. 14-Amputations. WHO amputations of lower limbs.

2. Abbo Toledano S. Preventive measures in the elderly amputee. Caracas, Medical Rehabilitation Bulletin. 1997;10(2):48-56.

3. Agreement of the National Group of Thermalism. Havana: Vice-Ministry of Medical Assistance, MINSAP; 1992.

4. Biermand Licht. Physical Medicine in General Practice. Hoeber; 1960.

5. Boldrey E. Amputation neuroma in nerves implanted in bone. Ann Surg. 1943;118(6):1052-1057.

6. Brunette D. The nature of phantom limb. J Tenn Med. 1993;73(10):712-773.

7. Runnstrom Kerr. Leg amputee: pre-prosthetic training.

8. José Antonio GH. Acceptance Therapy. 